\title{
Continuous Dependence on the Initial Condition of the Solution of a System of Differential Equations with Variable Structure and with Impulses
}

By

\author{
A. B. DishliEV* and D. D. BAINOV**
}

\section{Summary}

In the paper an initial value problem is considered for a system of ordinary differential equations with variable structure and impulses. Sufficient conditions are found under which there exists a solution of such system for $t \geq \tau_{0}$ where $\tau_{0}$ is a real constant Sufficient conditions are presented for continuous dependence of the solution of the initial condition.

\section{\$1. Introduction. Statement of the Problem}

In this paper we study dynamic processes that are subject to short time perturbations during their evolution, and as a result, the evolution law changes. The period of the perturbation effect can be neglected, therefore they are regarded as "momentary", i.e the perturbations are of an impulse type. Such processes are described by systems of ordinary differential equations with variable structure and with impulses.

The investigations of systems of differential equations with variable structure (without impulses) also called "systems with switchings", "tempestuous systems" etc. were originated in the works by T.Vogel $[1]-[5](1953)$ that have descriptive character. The theory of the variable structure systems finds its further development in the papers by A. Myshkis and Chohryakov [6] (1958), A. Myshkis and Parshikova [7] (1972) etc. In these systems there is a change in the right hand sides when the trajectory reaches give set defined in the phase space and called "critical set" (also "switching curves", "dis-

Communicated by S. Hitotumatu, July 28, 1986.

* Higher Institute of Chemical Technology, Sofia, Bulgaria.

** University of Plovdiv, Paisii Hilendarski, Plovdiv, Bulgaria. 
continuity curves" ets.). In the present paper the critical set consists of a countable set of hypersurfaces located in the extended phase space and there is a change of the structure when the integral curve meets some of these hypersurfaces.

The qualitative theory of systems with impulses (without variable structure) has been developed quite recently. The first papers in this field are V. Milman and A. Myshkis [8] (1960) and [9] (1963), A. Samoilenko [10] (1961) etc. In this paper we consider an initial value problem for a system of differential equations with variable structure and with impulses so that the impulses appear at the moment when the integral curve meets some of the given hypersurfaces. Such systems (without variable structure) are considered in [11].

The first paper in which systems of differential equations with variable structure and with impulses are considered is by D. Bainov and S. Milusheva [12] (1985). We note that in [12] the right hand side of the system is chosen among two functions.

Before the formulation of the problem considered in the paper we present a short description of these systems. We say that a system of differential equations with variable structure and impulses is given when the following set of mathematical objects and relations between them defined for every $i=0,1, \ldots$ is present:

1) A set of hypersurfaces

$$
\sigma_{i}: t=t_{i}(x), \quad t \in \mathbb{R}, \quad x \in D,
$$

where $D$ is a domain in $\boldsymbol{R}^{n}$.

2) A set of functions $\alpha_{i}: D \rightarrow \boldsymbol{R}$ called "switching functions".

3) A set of functions $I_{i}: D \rightarrow \boldsymbol{R}^{n}$ called "impulse functions" $I_{0}(x) \equiv 0, x \in D$.

4) A set of functions $f_{i}^{\alpha}: S \rightarrow \boldsymbol{R}^{n}, \alpha \in \boldsymbol{R}$, where $S=\left\{(t, x) ; t \geq \tau_{0}, x \in D\right\}$ 。

5) Systems of ordinary differential equations

$$
\frac{d x}{d t}=f_{s_{i}}^{\alpha}(t, x), \quad \tau_{i}<t \leq \tau_{i+j},
$$

where the points $\tau_{i}$ are the only moments at which the integral curve of the system of differential equations with variable structure and with impulses meets some of the hypersurfaces (1); $s_{i}$ is the index of the hypersurface which is met by the integral curve at the moment 
$\tau_{i}$, i. e. the equality $t_{s_{i}}\left(x\left(\tau_{i}\right)\right)=\tau_{i}, s_{0}=0$ holds (generally $i \neq s_{i}$ ); the parameter $\alpha$ is defined by the equality

$$
\alpha=\alpha_{s_{i}}\left(x\left(\tau_{i}\right)\right) \text {. }
$$

6) The equalities

$$
\left.\Delta x(t)\right|_{t=\tau_{i}}=x\left(\tau_{i}+0\right)-x\left(\tau_{i}\right)=I_{s_{i}}\left(x\left(\tau_{i}\right)\right),
$$

which describe the impulse perturbations.

This paper considers an initial value problem for the above system of differential equations with variable structure and impulses with the following initial condition

$$
x\left(\tau_{0}\right)=x_{0}, \quad x_{0} \in D .
$$

For convenience we introduce the notation:

$$
x_{i}=x\left(\tau_{i}\right), \quad x_{i}^{+}=x_{i}+I_{s_{i}}\left(x_{i}\right), \quad i=1,2, \ldots
$$

We shall describe the integral curve of the problem considered.

Let the point $P_{t}$ with current coordinates $(t, x(t))$ be the mapping point of the processes described by the problem (2)-(5). The mapping point starts its motion from the point $\left(\tau_{0}, x_{0}\right)$ and moves along the integral curve of the problem (2), (5) where $\alpha=\alpha_{0}\left(x_{0}\right)$ untill the moment $\tau_{1}$. At the moment $\tau_{1}$ the point $P_{t}$ meets the hypersurface $\sigma_{s_{1}}$ (we shall prove later on that $s_{1}=1$ ). At the moment $\tau_{1}$ it jumps momentary from the position $\left(\tau_{1}, x_{1}\right)$ to the position $\left(\tau_{1}, x_{1}^{+}\right)$and starts to move along the integral curve of the system (2) with initial condition $x\left(\tau_{1}+0\right)=x_{1}^{+}$where $\alpha=\alpha_{s_{1}}\left(x_{1}\right)$. The motion is continued till the moment $\tau_{2}$, at which the mapping point $P_{t}$ meets the hypersurface $\sigma_{s_{2}}$, where there is a new jump and a change of the motion law (i. e. a change of the right hand side of the system (2)) etc. If after a jump the point $P_{t}$ hits again the hypersurface from (1) then there is no second jump at this moment.

The curve, along which the point $P_{t}$ moves is called integral curve of the system of differential equations with variable structure and with impulses (more exactly the motion of the point $P_{t}$ described above is done along the integral curve of the problem (2)-(5)). The law according to which this motion is realize is called solution of the system. The solution is a piecewise continuous function with 
discontinuity point of the first type at which it is continuous from the left.

In the sequel we denote by $(t, x(t))$ the integral curve of the problem (2)-(5), and with $x\left(t, \tau^{*}, x^{*}\right)$ the solution of the system with variable structure and impulses with initial condition $x\left(\tau^{*}, \tau^{*}, x^{*}\right)=x^{*}$. Let $\|\cdot\|$ be the Euclidean norm in $\boldsymbol{R}^{n}$ and $\rho(A, B)$ be the Euclidean distance between the nonempty sets

$$
A, B \subset \boldsymbol{R}^{n}, \Omega_{i}=\left\{(t, x) ; t_{i-1}(x)<t<t_{i}(x), x \in D\right\}, i=1,2, \ldots
$$

Each constant greater than $\tau_{0}$ will be denoted by $T$.

Along with the problem (2)-(5) we consider the following initial value problem

$$
\begin{aligned}
& \frac{d x^{*}}{d t}=f_{m_{i}}^{\beta}\left(t, x^{*}\right), \quad \tau_{i}^{*}<t \leq \tau_{i+1}^{*}, \\
& \left.\Delta x^{*}(t)\right|_{t=\tau_{i}^{*}}=I_{m_{i}}\left(x^{*}\left(\tau_{i}^{*}\right)\right), \quad i=0,1, \ldots, \\
& x^{*}\left(\tau_{0}\right)=x_{0}^{*}, \quad x_{0}^{*} \in D,
\end{aligned}
$$

where $\tau_{i}^{*}, i=1,2, \ldots$ are the moments at which the integral curve $\left(t, x^{*}(t)\right)=\left(t, x\left(t, \tau_{0}^{*}, x_{0}^{*}\right)\right)$ meets hypersurfaces from (1), $\tau_{0}^{*}=\tau_{0}, m_{i}$ is the index of the hypersurface which is met by the integral curve $\left(t, x^{*}(t)\right)$ at the moment $\tau_{i}^{*}, m_{0}=0$ and $\beta=\alpha_{m_{i}}\left(x^{*}\left(\tau_{i}^{*}\right)\right)$.

As before we denote $x_{i}^{*}=x^{*}\left(\tau_{i}^{*}\right), x_{i}^{*+}=x_{i}^{*}+I_{m_{i}}\left(x_{i}^{*}\right), i=1,2, \ldots$

Definition 1. We say that the solution $x\left(t, \tau_{0}, x_{0}\right)$ of the problem (2) - (5) depends continuously on the initial conditions for $\tau_{0} \leq t \leq T$, if for every two positive numbers $\varepsilon$ and $\eta$ there exists a number $\delta=\delta(\varepsilon, \eta)>0$ such that if $\left\|x_{0}-x_{0}^{*}\right\|<\delta$ then $\left\|x\left(t, \tau_{0}, x_{0}\right)-x\left(t, \tau_{0}, x_{0}^{*}\right)\right\|<\varepsilon$ for $\tau_{0} \leq t \leq T$ and $\left|t-\tau_{1}\right|>\eta i=1,2, \ldots$

In the present paper sufficient conditions are obtained under which the solution $x\left(t, \tau_{0}, x_{0}\right)$ depends continuously on the initial conditions.

\section{§2. Auxiliary Results}

In the general case it is possible the integral curve to meet infinitely many times one and the same hypersurface from (1). This effect is called "beating". If beating appears it is possible the sequence 
of impulse moments to have a condensation point, i. e. the solution of the problem (2)-(5) to be not continuable for every $t>\tau_{0}$. Therefore it is necessary to require additional restrictive assumptions which eliminate the effect of beating.

We say that the conditions (A) are fulfilled if the following conditions hold for $i=1,2, \ldots$

Al. The functions $f_{\imath}^{\alpha}, \alpha \in \mathbb{R}$ are continuous in $S$ and Lipschitz continuous with respect to $x$ in $D$ uniformly in $t>\tau_{0}$ with respective constants $K_{i}$, which do not depend on $\alpha$.

A2. The following inequalities hold: $\left\|f_{i}^{\alpha}(t, x)\right\| \leq M_{i}$ for $(t, x) \in S$, $\alpha \in \mathbb{R}$, where the constants $M_{i}$ are positive and do not depend on $\alpha$.

A3. The integral curve $(t, x(t))$ of the problem (2)-(5) does not leave the set $I \times D$, where

$$
I= \begin{cases}{\left[\tau_{0},+\infty\right),} & \text { if the meetings of }(t, x(t)) \text { with the hypersurfaces } \\ & \text { are a finite number } r \\ {\left[\tau_{0}, a\right),} & \text { otherwise, } a=\lim _{i \rightarrow \infty} \tau_{i} .\end{cases}
$$

A4. The functions $t_{i}$ are Lipschitz continuous with respect to $x$ in $D$ with respective constants

$$
L_{i}<\min \left(1 / M_{i-1}, 1 / M_{i}\right), \quad M_{0}=M_{1} \text {. }
$$

A5. The following inequalities hold

$$
t_{i}\left(x+I_{i}(x)\right) \leq t_{i}(x), \quad x \in D .
$$

A6. For $x \in D$ the following inequalities hold

$$
\tau_{0}=t_{0}(x)<t_{1}(x)<t_{2}(x)<\ldots
$$

Theorem 1. Suppose that the conditions (A) hold. Then the integral curve $(t, x(t))$ meets each of the hypersurfaces at most once.

Proof. From the conditions $\mathrm{A} 1$ and $\mathrm{A} 3$ existence and uniqueness of the solution of the problem (2)-(5) follows for $t \in I$.

If the integral curve $(t, x(t))$ does not meet any hypersurface from (1) for $t \in I$, then the theorem is proved. Suppose that there is at least one meeting between $(t, x(t))$ and the hypersurfaces (1).

We shall show that $s_{i} \neq s_{i+1}, i=1,2, \ldots\left(s_{i}\right.$ is the index of the hypersurface which is met by the integral curve $(t, x(t))$ at the 
moment $\tau_{i}$ ). Assume the opposite, i. e. there exists an index $k$ such that $s_{k}=s_{k+1}$. Since for $\tau_{k}<t \leq \tau_{k+1}$ the solution of the problem (2)(5) coincides with the solution of the integral equation

$$
x(t)=x_{k}^{+}+\int_{\tau_{k}}^{t} f_{s_{k}}^{\alpha}(\tau, x(\tau)) d \tau
$$

then, according to the condition A2 for $t=\tau_{k+1}$ we get from (7)

$$
\left\|x_{k+1}-x_{k}^{+}\right\| \leq M_{s_{k}}\left(\tau_{k+1}-\tau_{k}\right) \text {. }
$$

Taking in consideration conditions A5 and A4 we find

$$
\begin{aligned}
\tau_{k+1}-\tau_{k} & =t_{s_{k+1}}\left(x_{k+1}\right)-t_{s_{k}}\left(x_{k}\right) \leq t_{s_{k}}\left(x_{k+1}\right)-t_{s_{k}}\left(x_{k}^{+}\right) \\
& \leq L_{s_{k}}\left\|x_{k+1}-x_{k}^{+}\right\|<\frac{1}{M_{s_{k}}}\left\|x_{k+1}-x_{k}\right\|,
\end{aligned}
$$

which contradicts (8).

We show that $s_{i}<s_{i+1}, i=1,2, \ldots$ Assume the opposite, i. e. there exists an index $k$ such that $s_{k}>s_{k+1}$. Let $\tau^{\prime}$ be an arbitrary point satisfying the inequalities $\tau_{k}<\tau^{\prime}<\tau_{k+1}$. Consider the function $\varphi(t)=$ $t-t_{s_{k}}(x(t))$ in the interval $\tau^{\prime} \leq t \leq \tau_{k+1}$. From the condition A6 we get

$$
\varphi\left(\tau_{k+1}\right)=\tau_{k+1}-t_{s_{k}}\left(x\left(\tau_{k+1}\right)\right)<\tau_{k+1}-t_{s_{k+1}}\left(x\left(\tau_{k+1}\right)\right)=0 .
$$

Using consequently the conditions A4 and A2 we find

$$
\begin{gathered}
\varphi\left(\tau^{\prime}\right)=\tau^{\prime}-t_{s_{k}}\left(x\left(\tau^{\prime}\right)\right)=\tau^{\prime}-\tau_{k}-\left(t_{s_{k}}\left(x\left(\tau^{\prime}\right)\right)-t_{s_{k}}\left(x\left(\tau_{k}\right)\right)\right. \\
>\tau^{\prime}-\tau_{k}-\frac{1}{M}\left\|x\left(\tau^{\prime}\right)-x_{k}^{+}\right\| \geq \tau^{\prime}-\tau_{k}-\left(\tau^{\prime}-\tau_{k}\right)=0
\end{gathered}
$$

From the inequalities (9) and (10) and since the function $\varphi$ is continuous for $\tau^{\prime} \leq t \leq \tau_{k+1}$ it follows that there exists a point $\tau^{\prime \prime}, \tau_{k}<\tau^{\prime}<$ $\tau^{\prime \prime}<\tau_{k+1}$ such that $\varphi\left(\tau^{\prime \prime}\right)=0$, i. e. $\tau^{\prime \prime}=t_{s_{k}}\left(x\left(\tau^{\prime \prime}\right)\right)$. Hence the integral curve $(t, x(t))$ meets the hypersurface $\sigma_{s_{k}}$ at the moment $\tau^{\prime \prime}$, which contradicts the method according to which the points $\tau_{i}$ are defined (see point 5 of the definition of a system with variable structure and with impulses).

Thus, Theorem 1 is proved.

We say that the condition (B) holds when the following condition is satisfied:

B. $\lim _{i \rightarrow \infty} t_{i}(x)=+\infty \quad$ uniformly in $x \in D$. 
Theorem 2. Suppose that the conditions (A) and (B) hold. Then the solution of the problem (2)-(5) is defined for $t \geq \tau_{0}$.

Proof. Since the solution of the problem considered is defined for $t \in I$, then if the moments $\tau_{i}$ are finite number the proof of the theorem is trivial. Suppose that the integral curve meets infinitely many hypersurfaces from (1). Then in order to prove the theorem it is sufficient to show that $\lim _{i \rightarrow \infty} \tau_{i}=+\infty$. According to Theorem 1 we have $s_{1}<s_{2}<\ldots$, i. e. $\lim _{\rightarrow \rightarrow \infty} s_{i}=+\infty$, hence, using the condition (B) we get

$$
\lim _{i \rightarrow \infty} \tau_{i}=\lim _{i \rightarrow \infty} t_{s_{i}}\left(x_{i}\right)=+\infty .
$$

This proves the theorem.

Theorem 3. Suppose that the conditions (A) hold and

$$
\left(\tau^{\prime}, x\left(\tau^{\prime}\right)\right) \in \Omega_{i}, \quad \tau^{\prime} \geq \tau_{0} .
$$

Then the integral curve $(t, x(t))$ meets at least one hypersurface of (1) meeting first the hypersurface $\sigma_{i \circ}$

Proof. Suppose that for $t>\tau^{\prime}$ the integral curve $(t, x(t))$ does not meet a hypersurface from (1). Then for $t>\tau^{\prime}$ the following inequality holds

$$
t<t_{i}(x(t)) \text {. }
$$

Really, if we suppose that there exists a point $\tau^{*}>\tau^{\prime}$ such that $\tau^{*}>t_{i}\left(x\left(\tau^{*}\right)\right)$, then for the function $\varphi(t)=t_{i}(x(t))-t$ defined for $t>\tau^{\prime}$ it holds $\varphi\left(\tau^{*}\right)<0$. Taking into account that the point $\left(\tau^{\prime}, x\left(\tau^{\prime}\right)\right) \in \Omega_{2}$ we get $\varphi\left(\tau^{\prime}\right)>0$. This implies that there exists a point $\tau \in\left[\tau^{\prime}, \tau^{*}\right]$ such that $\varphi(\tau)=0$. The latter means that the integral curve $(t, x(t))$ meets the hypersurface $\sigma_{i}$ at the moment $\tau$, which contradicts the supposition.

From (12) using the conditions A4 and A2 we obtain

$$
t-t_{i}\left(x\left(\tau^{\prime}\right)\right)<t_{i}(x(t))-t_{i}\left(x\left(\tau^{\prime}\right)\right)<L_{i} M_{i-1}\left(t-\tau^{\prime}\right),
$$

i. e. the inequalities hold

$$
t<\frac{t_{i}\left(x\left(\tau^{\prime}\right)\right)-L_{i} M_{i-1} \tau^{\prime}}{1-L_{i} M_{i-1}}=\theta=\text { const }
$$

The last inequality contradicts the fact that (12) holds for $t>\tau^{\prime}$ 
(containing the case $t>\theta$ ).

Suppose that the first hypersurface met by the integral curve $(t, x(t))$ for $t>\tau^{\prime}$ is $\sigma_{k}$ and the meeting realises at the moment $\tau^{\prime \prime}$, i. e.

$$
t_{k}\left(x\left(\tau^{\prime \prime}\right)\right)=\tau^{\prime \prime} \text {. }
$$

Suppose that $k<i-1$. Consider the function $\varphi(t)=t_{i-1}(x(t))-t$ defined in the interval $\tau^{\prime} \leq t \leq \tau^{\prime \prime}$. Since $\left(\tau^{\prime}, x\left(\tau^{\prime}\right)\right) \in \Omega_{i}$ then

$$
\varphi\left(\tau^{\prime}\right)<0 \text {. }
$$

From (13) and the condition A6 we obtain

$$
\varphi\left(\tau^{\prime \prime}\right)=t_{i-1}\left(x\left(\tau^{\prime \prime}\right)\right)-\tau^{\prime \prime}=t_{i-1}\left(x\left(\tau^{\prime \prime}\right)\right)-t_{k}\left(x\left(\tau^{\prime \prime}\right)\right)>0 .
$$

From the inequalities (14) and (15) and since the function $\varphi$ is continuous for $\tau^{\prime} \leq t \leq \tau^{\prime \prime}$ it follows that there exists a point $\tau^{\prime \prime}, \tau^{\prime}<\tau^{\prime \prime}<\tau^{\prime \prime}$ such that $\varphi\left(\tau^{*}\right)=0$ which contradicts the fact that the first hypersurface met by the integral curve $(t, x(t))$ for $t>\tau^{\prime}$ is $\sigma_{k}$.

Assume that $k>i$. Consider the function $\varphi(t)=t_{i}(x(t))-t$ defined in the interval $\tau^{\prime} \leq t \leq \tau^{\prime \prime}$. Analogously to (14) and (15) we find $\varphi\left(\tau^{\prime}\right)>0, \varphi\left(\tau^{\prime \prime}\right)<0$ from where we again come to a contradiction.

Suppose that $k=i-1$. Then from the condition A2 we get

$$
\left\|x\left(\tau^{\prime}\right)-x\left(\tau^{\prime \prime}\right)\right\|<M_{i-1}\left(\tau^{\prime \prime}-\tau^{\prime}\right) .
$$

From the condition A4 and from $\tau^{\prime}>t_{i-1}\left(x\left(\tau^{\prime}\right)\right)$ the following inequality holds

$$
\tau^{\prime \prime}-\tau^{\prime}<t_{i-1}\left(x\left(\tau^{\prime \prime}\right)\right)-t_{i-1}\left(x\left(\tau^{\prime}\right)\right)<\frac{1}{M_{i-1}}\left(\tau^{\prime \prime}-\tau^{\prime}\right),
$$

which contradicts (16).

This proves Theorem 3.

Corollary 1. If the conditions (A) hold then $s_{1}=1$.

\section{§3. Main Results}

We say that the conditions (C) are fulfilled if for $i=1,2, \ldots$ the following conditions are satisfied:

C1. The functions $I_{i}$ are continuous in $D$.

C2. The inequalities $t_{k}\left(x_{i}^{+}\right) \neq \tau_{i}, k=1,2, \ldots$ hold. 
C3. The functions $\alpha_{i}$ are continuous in $D$.

C4. The functions $f_{i}^{\alpha}$ depend continuously from the parameter $\alpha$ in $S$, i. e. for every positive number $\varepsilon$ there exists a number $\delta_{i}=\delta_{i}(\varepsilon)>0$ such that if $|\alpha-\beta|<\delta$ then $\left\|f_{i}^{\alpha}(t, x)-f_{i}^{\beta}(t, x)\right\|<\varepsilon$ uniformly in $S_{\text {。 }}$

Theorem 4. Suppose that the conditions (A) and (G) and the inequaliiies $\tau_{1}<T<\tau_{2}$ are satisfied.

Then for every two positive numbers $\varepsilon$ and $\eta$ there exists a positive number $\delta=\delta(\varepsilon, \eta)>0$ such that if $\left\|x_{0}-x_{0}^{*}\right\|<\delta$ then

a) $\left\|x(t)-x^{*}(t)\right\|<\gamma \varepsilon, \tau_{0} \leq t \leq T,\left|t-\tau_{1}\right|>\eta$;

b) $|\alpha-\beta|<\varepsilon$,

where $\gamma$ is a positive constant and $\alpha=\alpha_{1}\left(x_{1}\right), \beta=\alpha_{1}\left(x_{1}^{*}\right)$.

Proof. Let the inequality $\tau_{1} \leq \tau_{1}^{*}$ hold. We assume first that the inequalities $\tau_{1}^{*}<T<\tau_{2}^{*}$ hold (we prove later on that if the point $x_{0}^{*}$ is sufficiently close to $x_{0}$ then these inequalities are fulfilled). Let $\varepsilon$ and $\eta$ are arbitrary positive constants. For $\tau_{0} \leq t \leq \tau_{1}$ the functions $x$ and $x^{*}$ satisfy the following equations respectively

$$
x(t)=x_{0}+\int_{\tau_{0}}^{t} f_{0}^{\alpha}(\tau, x(\tau)) d \tau, \quad x^{*}(t)=x_{0}^{*}+\int_{\tau_{0}}^{t} f_{0}^{\beta}\left(\tau, x^{*}(\tau)\right) d \tau 。
$$

Substracting the sides for $t \in\left[\tau_{0}, \tau_{1}\right]$ we get the estimate

$$
\begin{aligned}
\left\|x(t)-x^{*}(t)\right\| & \leq\left\|x_{0}-x_{0}^{*}\right\|+\int_{\tau_{0}}^{t}\left\|f_{0}^{\alpha}(\tau, x(\tau))-f_{0}^{\alpha}\left(\tau, x^{*}(\tau)\right)\right\| d \tau \\
& +\int_{\tau_{0}}^{t}\left\|f_{0}^{\alpha}\left(\tau, x^{*}(\tau)\right)-f_{0}^{\beta}\left(\tau, x^{*}(\tau)\right)\right\| d \tau 。
\end{aligned}
$$

From the last inequality, taking into account the condition Al we get

$$
\begin{aligned}
\left\|x(t)-x^{*}(t)\right\| & \leq\left\|x_{0}-x_{0}^{*}\right\|+\int_{\tau_{0}}^{t} K_{0}\left\|x(\tau)-x^{*}(\tau)\right\| d \tau \\
& +\left(\tau_{1}-\tau_{0}\right) \sup _{\tau_{0} \leq \tau \leq \tau_{1}}\left\|f_{0}^{\alpha}\left(\tau, x^{*}(\tau)\right)-f_{0}^{\beta}\left(\tau, x^{*}(\tau)\right)\right\|_{0}
\end{aligned}
$$

From the condition C4 it follows that there exists a number $\delta_{1}=\delta_{1}(\varepsilon)>0$ such that for $|\alpha-\beta|<\delta_{1}$ the following inequality holds

$$
\sup _{\tau_{0} \leq \tau \leq \tau_{1}}\left\|f_{0}^{\alpha}\left(\tau, x^{*}(\tau)\right)-f_{0}^{\beta}\left(\tau, x^{*}(\tau)\right)\right\|<\varepsilon_{0}
$$

Since $\alpha=\alpha_{0}\left(x_{0}\right)$ and $\beta=\alpha_{0}\left(x_{0}^{*}\right)$ and taking advantage of the condition C3 we come to the conclusion that there exists a number $\delta_{2}=\delta_{2}\left(\delta_{1}\right)>0$ 
such that if $\left\|x_{0}-x_{0}^{*}\right\|<\delta_{2}$ then $|\alpha-\beta|<\delta_{1}$. Let $\delta_{3}=\min \left(\varepsilon, \delta_{2}\right)$. Then, if $\left\|x_{0}-x_{0}^{*}\right\|<\delta_{3}$ then (18) holds and the following inequality holds as well

$$
\left\|x_{0}-x_{0}^{*}\right\|<\varepsilon .
$$

From (17), (18) and (19) we get

$$
\left\|x(t)-x^{*}(t)\right\| \leqq \varepsilon+\left(\tau_{1}-\tau_{0}\right) \varepsilon+\int_{\tau_{0}}^{t} K_{0}\left\|x(\tau)-x^{*}(\tau)\right\| d \tau, \quad \tau_{0} \leq t \leq \tau_{1} .
$$

Using the Bellman-Gronwall inequality we find the estimate

$$
\begin{aligned}
\left\|x(t)-x^{*}(t)\right\| & \leq \varepsilon\left(1+\tau_{1}-\tau_{0}\right) \exp \left(K_{0}\left(\tau_{1}-\tau_{0}\right)\right) \\
& \leq \varepsilon(1+T) \exp \left(K_{0} T\right)=\gamma_{1} \varepsilon, \quad \tau_{0} \leq t \leq \tau_{1} .
\end{aligned}
$$

According to Corollary $1 s_{1}=m_{1}=1$. Hence

$$
\begin{aligned}
\left|\tau_{1}-\tau_{1}^{*}\right| & \leq\left|\tau_{1}-t_{1}\left(x^{*}\left(\tau_{1}\right)\right)\right|+\mid t_{1}\left(x^{*}\left(\tau_{1}\right)-\tau_{1}^{*} \mid\right. \\
& =\left|t_{1}\left(x_{1}\right)-t_{1}\left(x^{*}\left(\tau_{1}\right)\right)\right|+\left|t_{1}\left(x^{*}\left(\tau_{1}\right)\right)-t_{1}\left(x_{1}^{*}\right)\right| .
\end{aligned}
$$

From the last inequality, the conditions A2 and A4 and from the inequality (20) for $t=\tau_{1}$ we obtain

$$
\begin{aligned}
\left|\tau_{1}-\tau_{1}^{*}\right| & \leq L_{1}|| x\left(\tau_{1}\right)-x^{*}\left(\tau_{1}\right)\left\|+L_{1}|| x *\left(\tau_{1}\right)-x^{*}\left(\tau_{1}^{*}\right)\right\| \\
& \leq L_{1} \gamma_{1} \varepsilon+L_{1} M_{0}\left|\tau_{1}-\tau_{1}^{*}\right|,
\end{aligned}
$$

which yields the following estimate

$$
\left|\tau_{1}-\tau_{1}^{*}\right|<\frac{L_{1} \gamma_{1}}{1-L_{1} M_{0}} \varepsilon .
$$

From the inequalities (20) and (21) we obtain the estimate

$$
\begin{aligned}
\left\|x_{1}-x_{1}^{*}\right\| & \leq\left\|x_{1}-x^{*}\left(\tau_{1}\right)\right\|+\left\|x^{*}\left(\tau_{1}\right)-x^{*}\left(\tau_{1}^{*}\right)\right\| \\
& \leq \gamma_{1} \varepsilon+M_{0}\left|\tau_{1}-\tau_{1}^{*}\right|=\frac{\gamma_{1}}{1-L_{1} M_{0}} \varepsilon_{0}
\end{aligned}
$$

Using the inequality (22) we get

$$
\begin{aligned}
\left\|x_{1}^{+}-x_{1}^{*+}\right\| & \leq\left\|x_{1}-x_{1}^{*}\right\|+\left\|I_{1}\left(x_{1}\right)-I_{1}\left(x_{1}^{*}\right)\right\| \\
& \leq \frac{\gamma_{1}}{1-L_{1} M_{0}} \varepsilon+\left\|I_{1}\left(x_{1}\right)-I_{1}\left(x_{1}^{*}\right)\right\|_{。}
\end{aligned}
$$

According to the condition $\mathrm{Cl}$ there exists a number $\delta_{4}>0$ such that if $\left\|x_{1}-x_{1}^{*}\right\|<\delta_{4}$ then

$$
\left\|I_{1}\left(x_{1}\right)-I_{1}\left(x_{1}^{*}\right)\right\|<\varepsilon .
$$

From the inequality (22) it is seen that there exists a number $\delta_{5}=\delta_{5}\left(\delta_{4}\right)>0$ such that if $\left\|x_{0}-x_{0}^{*}\right\|<\delta_{5}$ then $\left\|x_{1}-x_{1}^{*}\right\|<\delta_{4}$ which yields 
(24). Then from (23) and (24) we get the estimate

$$
\left\|x_{1}^{+}-x_{1}^{*+}\right\| \leq \frac{1+\gamma_{1}-L_{1} M_{0}}{1-L_{1} M_{0}} \varepsilon .
$$

From the inequalities (21) and (25) we conclude that

$$
\begin{aligned}
\left\|x\left(\tau_{1}^{*}\right)-x_{1}^{*+}\right\| & \leq\left\|x\left(\tau_{1}^{*}\right)-x_{1}^{+}\right\|+\left\|x_{1}^{+}-x_{1}^{*+}\right\| \\
& \leq \frac{L_{1} M_{1} \gamma_{1}}{1-L_{1} M_{0}} \varepsilon+\frac{1+\gamma_{1}-L_{1} M_{0}}{1-L_{1} M_{0}} \varepsilon=\gamma_{2} \varepsilon_{0}
\end{aligned}
$$

The functions $x, x^{*}$ satisfy the equations in the interval $\tau_{1}^{*}<t \leq T$ :

$$
x(t)=x\left(\tau_{1}^{*}\right)+\int_{\tau_{1}^{*}}^{t} f_{1}^{\alpha}(\tau, x(\tau)) d \tau, x^{*}(t)=x_{1}^{*+}+\int_{\tau_{1}^{*}}^{t} f_{1}^{\beta}\left(\tau, x^{*}(\tau)\right) d \tau,
$$

which imply

$$
\begin{aligned}
\left\|x(t)-x^{*}(t)\right\| & \leq\left\|x\left(\tau_{1}^{*}\right)-x_{1}^{*+}\right\|+\int_{\tau_{1}^{*}}^{t}\left\|f_{1}^{\alpha}(\tau, x(\tau))-f_{1}^{\alpha}\left(\tau, x^{*}(\tau)\right)\right\| d \tau \\
& +\int_{\tau_{1}^{*}}^{t}\left\|f_{1}^{\alpha}\left(\tau, x^{*}(\tau)\right)-f_{1}^{\beta}\left(\tau, x^{*}(\tau)\right)\right\| d \tau_{0}
\end{aligned}
$$

Using the estimate (26) and the condition Al we find

$$
\begin{aligned}
& \left\|x(t)-x^{*}(t)\right\| \leq \gamma_{2} \varepsilon+\int_{\tau_{1}^{*}}^{t} K_{1}\left\|x(\tau)-x^{*}(\tau)\right\| d \tau \\
& \quad+\left(T-\tau_{1}^{*}\right) \sup _{\tau_{1}^{*} \leq \tau \leq T}\left\|f_{1}^{\alpha}\left(\tau, x^{*}(\tau)\right)-f_{1}^{\beta}\left(\tau, x^{*}(\tau)\right)\right\|, \tau_{1}^{*}<t \leq T .
\end{aligned}
$$

According to the condition C4 there exists a number $\delta_{6}=\delta_{6}(\varepsilon)>0$ such that for $|\alpha-\beta|<\delta_{6}$,

$$
\sup _{\tau_{1} \leq \tau \leq T}\left\|f_{1}^{\alpha}\left(\tau, x^{*}(\tau)\right)-f_{1}^{\beta}\left(\tau, x^{*}(\tau)\right)\right\|<\varepsilon_{0}
$$

If the number $\left\|x_{0}-x_{0}^{*}\right\|$ is sufficiently small then from (22) it follows that the number $\left\|x_{1}-x_{1}^{*}\right\|$ can become smaller than an arbitrarily fixed positive constant. Taking into account that in the equalities (27) $\alpha=\alpha_{1}\left(x_{1}\right)$ and $\beta=\alpha_{1}\left(x_{1}^{*}\right)$ then from C3 it follows that there exists a number $\delta_{7}=\delta_{7}\left(\delta_{6}\right)>0$ such that if $\left\|x_{0}-x_{0}^{*}\right\|<\delta_{7}$ then $|\alpha-\beta|<\delta_{6}$. The last conclusion implies (29). From (28), (29) and the GronwallBellman inequality we obtain the estimate

$$
\begin{aligned}
\left\|x(t)-x^{*}(t)\right\| & \leq \varepsilon\left(\gamma_{2}+T-\tau_{1}^{*}\right) \exp \left(K_{1}\left(T-\tau_{1}^{*}\right)\right) \\
& \leq \varepsilon\left(\gamma_{2}+T\right) \exp \left(K_{1} T\right)=\gamma_{3} \varepsilon, \quad \tau_{1}^{*}<t \leq T .
\end{aligned}
$$

From the inequalities (20) and (30) it follows that if the number $\left\|x_{0}-x_{0}^{*}\right\|<\delta_{8}=\min \left(\delta_{1}, \delta_{2}, \ldots, \delta_{7}\right)$ then 


$$
\left\|x(t)-x^{*}(t)\right\| \leq \gamma \varepsilon, \quad \tau_{0} \leq t \leq \tau_{1}, \quad \tau_{1}^{*}<t \leq T, \gamma=\max \left(\gamma_{1}, \gamma_{3}\right) .
$$

Taking into account the inequality (19) we come to the conclusion that there exists a number $\delta_{9}>0$ such that if $\left\|x_{0}-x_{0}^{*}\right\|<\delta_{9}$ then $\left|\tau_{1}-\tau_{1}^{*}\right|<\eta_{0}$. The last inequality and (31) imply

$$
\left\|x(t)-x^{*}(t)\right\| \leq \gamma \varepsilon, \quad \tau_{0} \leq t \leq T, \quad\left|t-\tau_{1}\right|>\eta .
$$

Finally we show that the assumption in the begining of the proof that $\tau_{1}^{*}<T<\tau_{2}^{*}$ is not essential. Really, from the inequality (21) and since $\tau_{1}<T<\tau_{2}$ if the number $\left\|x_{0}-x_{0}^{*}\right\|$ is sufficiently small the inequality holds $\tau_{1}^{*}-\tau_{1}<T-\tau_{1}$, i. e. $\tau_{1}^{*}<T$ (We note that in the proof of (21) the assumtion from the begining of the proof is not used). Taking into account that the set $A=\mathbb{R}^{n+1} \backslash \Omega_{s_{2}}$ is closed and the set $B=\left\{(t, x) ; x=x(t), \tau_{1}^{*}<t \leq T\right\}$ is closed and bounded and $A \cap B=\phi$ we conclude that $\rho(A, B)=\delta_{10}>0$.

Assume that $\tau_{2}^{*}<T$. Then analogously to (30) we get the estimate

$$
\begin{aligned}
\left\|x(t)-x^{*}(t)\right\| & \leq \varepsilon\left(\gamma_{2}+\tau_{2}^{*}-\tau_{1}^{*}\right) \exp \left(K_{1}\left(\tau_{2}^{*}-\tau_{1}^{*}\right)\right) \\
& \leq \gamma_{3} \varepsilon, \quad \tau_{1}^{*}<t \leq \tau_{2}^{*} .
\end{aligned}
$$

The last inequality implies that if the number $\varepsilon$ is sufficiently small then $\left\|x\left(\tau_{2}^{*}\right)-x^{*}\left(\tau_{2}^{*}\right)\right\|<\delta_{10}$ which yields that the point $\left(\tau_{2}^{*}, x_{2}^{*}\right) \in \Omega_{s_{2}}$. Hence the integral curve $\left(t, x^{*}(t)\right)$ does not meet a hypersurface from (1) at the moment $\tau_{2}^{*}$. The last conclusion contradicts the manner on which the points $\tau_{i}^{*}$ are defined. Hence the inequality $\tau_{2}^{*}>T$ holds.

Let $\tau_{1}>\tau_{1}^{*}$. We show that if the point $x_{0}^{*}$ is sufficiently close to the point $x_{0}$ then the integral curve $\left(t, x^{*}(t)\right)$ does not meet a hypersurface from (1) for $\tau_{1}^{*}<t<\tau_{1}$. Clearly, since the point $\left(\tau_{1}, x_{1}^{+}\right)$does not belong to the closed set $A$ then

$$
\rho\left(\left(\tau_{1}, x_{1}^{+}\right), A\right)=\varepsilon_{1}>0 .
$$

Analogously to (21) and (25) we obtain the estimates

$$
\tau_{1}-\tau_{1}^{*}<\frac{L_{1} \gamma_{1}}{1-L_{1} M_{0}} \varepsilon, \quad\left\|x_{1}^{+}-x_{1}^{*+}\right\|<\frac{1+\gamma_{1}-L_{1} M_{0}}{1-L_{1} M_{0}} \varepsilon .
$$

Assume that for $\tau_{1}^{*}<t<\tau_{1}$ the integral curve $\left(t, x^{*}(t)\right)$ meets a hypersurface from (1), i. e. it holds $\tau_{2}^{*}<\tau_{1}$. Then taking into account that the function $x^{*}$ for $\tau_{1}^{*}<t \leq \tau_{2}^{*}$ satisfies the second of the equations (27) we find the estimate

$$
\left\|x_{2}^{*}-x_{1}^{*+}\right\| \leq M_{1}\left(\tau_{2}^{*}-\tau_{1}\right)<M_{1}\left(\tau_{1}-\tau_{1}^{*}\right)<\frac{M_{1} L_{1} \gamma_{1}}{1-L_{1} M_{0}} \varepsilon_{。}
$$


We estimate the distance between the points $\left(\tau_{1}, x_{1}^{+}\right)$and $\left(\tau_{2}^{*}, x_{2}^{*}\right)$,

$$
\begin{aligned}
\rho\left(\left(\tau_{1}, x_{1}^{+}\right),\left(\tau_{2}^{*}, x_{2}^{*}\right)\right) & \leq\left(\tau_{1}-\tau_{1}^{*}\right)+\left\|x_{1}^{+}-x_{1}^{*+}\right\|+\left\|x_{1}^{*+}-x_{2}^{*}\right\| \\
& <\frac{L_{1} \gamma_{1}+1+\gamma_{1}-L_{1} M_{0}+M_{1} L_{1} \gamma_{1}}{1-L_{1} M_{0}} \varepsilon_{\circ}
\end{aligned}
$$

From the last inequality it follows that if the point $\varepsilon$ is sufficiently small which means that the point $x_{0}^{*}$ is close to the point $x_{0}$ then the following inequality holds

$$
\rho\left(\left(\tau_{1}, x_{1}^{+}\right),\left(\tau_{2}^{*}, x_{2}^{*}\right)\right)<\varepsilon_{1} .
$$

The last inequality means that the point $\left(\tau_{2}^{*}, x_{2}^{*}\right) \in \Omega_{s_{2}}$ i. e. it does not belong to a hypersurface from (1). This contradicts the manner according to which the moments $\tau_{\imath}^{*}$ are defined. Hence the integral curve $\left(t, x^{*}(t)\right)$ does not meet a hypersurface from (1) for $\tau_{1}^{*}<t \leq \tau_{1}$ 。 The further considerations are analogous to the case $\tau_{1} \leq \tau_{1}^{*}$.

Thus, Theorem 4 is proved.

From Theorem 4 we obtain the following corollary:

Corollary 2. Let the conditions (A) and (G) hold and there exist a number $K$ such that $\tau_{k-1}<t_{1}<\tau_{k}<t_{2}<\tau_{k+1}$ 。

Then for every two positive numbers $\varepsilon, \eta$ there exists a point $\delta=\delta(\varepsilon, \eta)>0$ such that if $\left\|x\left(t_{1}\right)-x^{*}\left(t_{1}\right)\right\|<\delta$ and $\left|\alpha_{s_{k}}\left(x_{k}\right)-\alpha_{s_{k}}\left(x_{k}^{*}\right)\right|<\delta$ then:

a) $\left\|x(t)-x^{*}(t)\right\|<\gamma \varepsilon, \quad t_{1} \leq t \leq t_{2}, \quad\left|t-\tau_{k}\right|>\eta$;

b) $\left|\alpha_{s_{k+1}}\left(x_{k+1}\right)-\alpha_{s_{k+1}}\left(x_{k+1}^{*}\right)\right|<\varepsilon$;

c) The integral curve $\left(t, x^{*}(t)\right)$ for $t_{1} \leq t \leq t_{2}$ meets the hypersurface $\sigma_{s_{k}}$ only where $\gamma$ is a positive constant.

Our principal result of the work follows:

Theorem 5. Suppose that the conditions (A), (B) and (C) hold. Then the solution of the problem (2)-(5) depends continuously on the initial condition for $\tau_{0} \leq t \leq T$.

Proof. According to the equality (11) $\lim _{i \rightarrow \infty} \tau_{i}=+\infty$ from which follows that for $\tau_{0} \leq t \leq T$ the integral curve $(t, x(t))$ meets a finite number of hypersurfaces of (1) i. e. $\tau_{0}<\tau_{1}<\ldots<\tau_{p} \leq T<\tau_{p+1}$. Introduce the notation $t_{i}=\left(\tau_{i}+\tau_{i+1}\right) / 2, i=1,2, \ldots, p-1, t_{0}=\tau_{0}, t_{p}=T$. Let $\varepsilon$ and $\eta$ are arbitrary positive numbers.

According to Corollary 2 there exists a number $\delta_{1}>0$ such that 
if $\left\|x_{0}-x_{0}^{*}\right\|<\delta_{1}$ then: 1) the integral curve $\left(t, x^{*}(t)\right)$ for $t_{0} \leq t \leq t_{1}$ meets the hypersurface $\sigma_{1}$ only and once only; 2) the inequality holds $\left\|x(t)-x^{*}(t)\right\|<\varepsilon$ for $\left.t_{0} \leq t \leq t_{1}, \quad\left|t-\tau_{1}\right|>\eta ; 3\right)$ the inequalities hold $\left\|x\left(t_{1}\right)-x^{*}\left(t_{1}\right)\right\|<\delta_{2}$ and $\left|\alpha_{1}\left(x_{1}\right)-\alpha_{1}\left(x_{1}^{*}\right)\right|<\delta_{2}$ where the constant $\delta_{2}$ will be specified further on. According to Corollary 2 there exists a number $\delta_{2}>0$ such that if $\left\|x\left(t_{1}\right)-x^{*}\left(t_{1}\right)\right\|<\delta_{2}$ and $\left|\alpha_{1}\left(x_{1}\right)-\alpha_{1}\left(x_{1}^{*}\right)\right|<\delta_{2}$ then : 1) the integral curve $\left(t, x^{*}(t)\right)$ for $t_{1} \leq t \leq t_{2}$ meets the hypersurface $\sigma_{s_{2}}$ only (that one which is met by $\left(t, x(t)\right.$ ) at the moment $\tau_{2}$ as well) and once only; 2) The inequality holds $\left\|x(t)-x^{*}(t)\right\|<\varepsilon$ for $\left.t_{1} \leq t \leq t_{2}, \quad\left|t-\tau_{2}\right|>\eta ; 3\right)$ the inequalities hold $\left\|x\left(t_{2}\right)-x^{*}\left(t_{2}\right)\right\|<\delta_{3}$ and $\left|\alpha_{s_{2}}\left(x_{2}\right)-\alpha_{s_{2}}\left(x_{2}^{*}\right)\right|<\delta$ where $\delta_{3}$ will be specified further on etc.

We set $\delta_{p+1}=\varepsilon$ and determine the numbers $\delta_{p}, \delta_{p-1}, \ldots, \delta_{1}$ sequentially so that the above conditions to be satisfied. Let $\delta=\min \left(\delta_{1}, \delta_{2}, \ldots, \delta_{p}\right)$. Then if $\left\|x_{0}-x_{0}^{*}\right\|<\delta$ then the inequality holds $\left\|x(t)-x^{*}(t)\right\|<\varepsilon$ for $\tau_{0} \leq t \leq T$ and $\left|t-\tau_{i}\right|>\eta_{\text {。 }}$

Theorem 5 is proved.

\section{References}

[1] Vogel, T., Sur les systemes deferelants, Bull. Soc. Math. France, 81: 1 (1953), 63-75.

[2] - Systemes dynamiques hereditaires a deferlement, Rend. Seminar math. Univ. Padove, 22: 1 (1953), 64-80.

[3] - Systemes dynamiques hereditaires a deferlement, Ann. telecomuns, 8:11 (1953), 354-360.

[4] Topologie des oscollations a deferlement, Actes colloq. Internat. Vibrations nonlineares. Ile de Porquerolles (1951), 237-256, Publ. Sci. Techn. Ministere de l'Air, Paris, 281.

[5] - Verifications experimentales de la theorie des systemes deferlants, Journ. Phis et radium, 14:12 (1953), 59.

[6] Myshkis, A. D., A. Y. Chohryakov, Future dynamical systems. I. Singular points on the plane, Math. sbornik, $45: 3$ (1958), 400-414 (russ.)

[7] Myshkis, A. D., N.P, Parshikova, Oscillations in systems with switchings, 6-th International Conf. on Nonlinear Oscill. Summaries, Warsaw, 1972, 94-95. (russ.)

[8] Milman, V.D., A. D. Myshkis, On the stability of motion in presence of impulses, Sib. Math. J., $1: 2$ (1960), 233-237. (russ.)

[9] , Random impulses in the linear dynamical systems, Asymptotic methods for solving differential equations, Izdat. Ac. Sc. USSR, Kiev (1963), 64-81 (russ.)

[10] Samoilenko, A. M., Application of the averaging method in the investigations of oscollations generated by impulses in the auto oscillation system of 2 nd order with a small parameter, UMJ, 13 No. 3 (1961).

[11] Samoilenko, A. M., N. A. Perestiuk, Stability of solutions of differential equations with impulse effect, Diff. Equations, 11 (1977), 1981-1992.

[12] Bainov, D.D., S. D. Milusheva, Investigation of partially-multiplicative averaging for a class of functional-differential equations with variable structure and impulses, Tsukuba J. Math, $9: 1$ (1985), 1-19. 